\title{
Addressing ethical challenges in HIV prevention research with people who inject drugs
}

\author{
Liza Dawson, ${ }^{1}$ Steffanie A Strathdee, ${ }^{2}$ Alex John London, ${ }^{3}$ Kathryn E Lancaster, ${ }^{4}$ \\ Robert Klitzman, ${ }^{5}$ Irving Hoffman, ${ }^{4}$ Scott Rose, ${ }^{6}$ Jeremy Sugarman ${ }^{7}$
}

'Division of AIDS, NIH/NIAID, Bethesda, Maryland, USA ${ }^{2}$ University of San Diego, San Diego, California, USA

${ }^{3}$ Carnegie Mellon University, Pittsburgh, Pennsylvania, USA ${ }^{4}$ University of North Carolina, Chapel Hill, North Carolina, USA ${ }^{5}$ Columbia University, New York, New York, USA

${ }^{6}$ Family Health International, Durham, North Carolina, USA ${ }^{7} J$ ohns Hopkins University, Baltimore, Maryland, USA

Correspondence to

Dr Liza Dawson, Division of AIDS, NIH/NIAID, 5601 Fisher's Lane, Room 9G48, Bethesda, MD 20892, USA:

dawson|@niaid.nih.gov

Received 14 May 2015 Revised 23 March 2016 Accepted 4 April 2016 Published Online First 25 April 2016

\section{Linked}

- http://dx.doi.org/10.1136/ medethics-2016-103571 - http://dx.doi.org/10.1136/ medethics-2016-103572 - http://dx.doi.org/10.1136/ medethics-2016-103573

- http://dx.doi.org/10.1136/ medethics-2017-104591

Check for updates

To cite: Dawson L

Strathdee SA, London AJ,

et al. J Med Ethics

2018:44:149-158.

\section{ABSTRACT}

Despite recent advances in HIV prevention and treatment, high HIV incidence persists among people who inject drugs (PWID). Difficult legal and political environments and lack of services for PWID likely contribute to high HIV incidence. Some advocates question whether any HIV prevention research is ethically justified in settings where healthcare system fails to provide basic services to PWID and where implementation of research findings is fraught with political barriers. Ethical challenges in research with PWID include concern about whether research evidence will be translated into practice; concerns that research might exacerbate background risks; and ethical challenges regarding the standard of HIV prevention in research. While these questions arise in other research settings, for research with PWID, these questions are especially controversial. This paper analyses four ethical questions in determining whether research could be ethically acceptable: (1) Can researchers ensure that research does not add to the burden of social harms and poor health experienced by PWID? (2) Should research be conducted in settings where it is uncertain whether research findings will be translated into practice? (3) When best practices in prevention and care are not locally available, what standard of care and prevention is ethically appropriate? (4) Does the conduct of research in settings with oppressive policies constitute complicity? We outline specific criteria to address these four ethical challenges. We also urge researchers to join the call to action for policy change to provide proven safe and effective HIV prevention and harm reduction interventions for PWID around the world.

\section{INTRODUCTION}

Significant advances in preventing, diagnosing and treating HIV have contributed to the global decline of HIV incidence and mortality. ${ }^{1}$ Access to evidence-based HIV prevention, testing and counselling, and treatment and care services have expanded. Despite these achievements, HIV incidence among people who inject drugs (PWID) continues to increase in many regions. HIV infection among PWID is driving HIV epidemics in Eastern Europe, the Commonwealth of Independent States, Central and South-East Asia, and the Middle East and North Africa. ${ }^{2}$ Global estimates suggest that 15.9 million people might inject drugs. ${ }^{2}$ An estimated three million PWID are likely to be HIV-infected, comprising $5-10 \%$ of all people living with HIV globally and 30\% outside sub-Saharan Africa. ${ }^{1}{ }^{3}$ The persistently high HIV incidence and prevalence among PWID is a substantial global health concern.
Due to structural factors that operate at multiple levels, as described below, HIV-infected PWID are often not engaged in care, leading to poor antiretroviral therapy (ART) uptake and adherence, increased HIV transmission, and the likely transmission of drug-resistant strains. ${ }^{2}$ The broad adoption of evidence-based strategies would help mitigate HIV risks among PWID, yet their adoption can be obstructed by deeply entrenched structural and policy factors. At the same time, researchers are actively attempting to improve the evidence base for appropriate care and treatment for PWID in spite of the structural barriers to effective care.

Some commentators and advocates have challenged the idea that HIV prevention research should be carried out at all in populations experiencing multiple levels of social, legal or political disadvantage. First, is conducting further research a reasonable means of addressing the health needs of PWID? Some advocates for civil rights, legal protections and better services for PWID view clinical research as a distraction from these broader struggles; or worse, they may view research efforts as an attempt to ignore or whitewash oppressive practices. ${ }^{4}$ Therefore the first challenge is to consider whether or under what conditions research would be an effective and acceptable mechanism to advance the health and welfare of PWID. Second, if research has a valuable role to play, are there populations of PWID that experience relatively little oppression, whose participation in research is less ethically controversial? Could research objectives be reasonably accomplished with populations that are less burdened with social and legal difficulties? Would this be ethically preferable? Finally, if research does go forward, how should it be designed and conducted?

In this paper, we first review briefly the background conditions that can contribute to HIV risk among PWIDs. We then analyse four key ethical questions on the issues of vulnerability, responsiveness and reasonable availability, standard of care and prevention, and complicity, all affecting the conduct of HIV prevention and treatment research with PWID. Based on this analysis, we provide criteria for responding to these challenges with minimum requirements that include meaningful, inclusive and robust community engagement as well as strict criteria for providing benefit and mitigating the risks faced by PWID in many settings. We suggest, furthermore, that researchers join the call to action to address flawed policies denying proven effective health interventions to PWID, and that all researchers, including those conducting 
biomedical prevention trials, join the effort to ensure delivery of the globally accepted package of prevention interventions for PWID in all settings where research is conducted.

\section{BACKGROUND CONDITIONS}

Interventions such as opioid substitution therapy (OST), syringe exchange programmes (SEPs) and expanded use of ART can significantly curtail HIV transmission among PWIDs and are considered essential components of a successful combination prevention programme for PWID. ${ }^{5}$ Furthermore, methadone and buprenorphine maintenance are considered essential medicines by WHO, but coverage of these interventions is extremely low. Worldwide, an estimated two syringes were distributed per PWID per month although most PWID inject at least twice per day. Coverage of OST was estimated at 8 recipients per 100 PWID in 2008, with wide variations between countries. Only about $4 \%$ of all HIV-infected PWID are on ART. ${ }^{3}$

HIV risk reduction and prevention modalities that have been developed with non-drug using populations, such as preexposure prophylaxis and HIV treatment as prevention, may be difficult to implement with PWID, due to social and structural conditions that inhibit access to services. A large scale-up of HIV prevention, treatment and care for PWID would undoubtedly help control the epidemic in this population; however, some policymakers and healthcare providers may be reluctant to encourage or facilitate access to care for PWID owing to fears or addictophobia-namely, fear, aversion and discrimination with regard to PWID. ${ }^{6}$ Efforts to improve access are often undermined by multiple barriers including social marginalisation and criminalisation of injection drug use, resulting in inadequate worldwide coverage of services for PWID. ${ }^{3} 78$ We emphasise that biomedical HIV treatment and prevention interventions are not a substitute for harm reduction approaches, but are a critical component of healthcare systems that should be available to all individuals at risk.

Global disparities in access to HIV prevention services for PWID may be explained at least in part by structural barriers, which include laws governing substance use, OST and syringe purchase and possession. For example, in 12 countries, drug and alcohol offences are punishable by judicial corporal punishment, which is a violation of international law. ${ }^{9}$ In Russia, OST is illegal. ${ }^{10}$ Several countries (eg, China, Mexico, Thailand and Vietnam) continue to operate compulsory treatment programmes (eg, forced labour or military training camps) which do not provide OST. Such programmes have not been effective in reducing drug use, and have been associated with higher HIV risk behaviours and other harms that constitute human rights violations. ${ }^{11}{ }^{12}$ While other countries avoid such punitive measures, some such as the USA have laws that prohibit the purchase of syringes without a prescription, or prohibit syringe exchange, which limits PWID's' access to sterile injection equipment. Even in settings where syringe access is permitted, PWID carrying used syringes can still be charged with drug possession due to trace amounts of illicit drugs in the used syringes that can be used as evidence. ${ }^{13}$

Policing practices are perhaps the most pervasive structural barrier that undermines HIV prevention for PWID. Arrests for drug possession, soliciting bribes and confiscating syringes have consistently been shown to influence where, with whom, when and how PWID administer their doses. Policing practices can directly affect PWID by pressuring them to inject hurriedly in the street, in shooting galleries where needles are rented or sold, or by seeking out 'hit doctors' who assist PWID with injection, usually in exchange for splitting the shared drug solution. These practices increase the risk of needle sharing, transmission of HIV and other bloodborne infections, and overdose mortality. ${ }^{13}$ Police misconduct, such as physical and sexual abuse perpetrated against PWID is common. In Thailand, 38\% of PWID were beaten by police, which was associated with higher odds of syringe sharing and reduced access to healthcare. ${ }^{14}$ It has been estimated that if police beatings were eliminated in Odessa, Ukraine, HIV incidence among PWID would decrease up to $19 \%$ over a 5 -year period. ${ }^{15}$ Policing can also indirectly increase transmission of HIV and bloodborne infections by discouraging PWID from carrying syringes, using SEPs or displacing PWID to areas with limited access to SEPs or OST. In the USA, SEPs reported the following police interactions at least monthly: client harassment: 43\%; confiscation of clients' syringes: 31\%, client arrest: $12 \% .^{16}$ In Ukraine, police harassment is a major barrier to use of SEPs, OST and even ART adherence among PWID. ${ }^{17}$ In some countries in Eastern Europe, Central and South-East Asia, PWID are 'registered' with authorities, which contributes to stigma and avoidance of HIV prevention services.

\section{VULNERABILITY: CAN THE RIGHTS AND WELFARE OF PARTICIPANTS IN CLINICAL TRIALS BE PROTECTED?}

International ethics guidelines stipulate that populations that are vulnerable should only be enrolled in research if equivalent research cannot be conducted with non-vulnerable groups. In many cases, vulnerability refers to impairment in ability to provide free and informed consent to research-hence, the ethical argument to prefer enrolment of non-vulnerable groups is based on the fundamental importance of consent. ${ }^{18}{ }^{19}$ For example, individuals with impairment in decision-making capacity, or children, may need special protections of this type.

Discussions of vulnerability and research protection can become confused by the multiple meanings of the word vulnerable. $^{2021}$ The concept of vulnerability can be broader than the ability to decide or consent, and can refer instead to economic or social disadvantage or risk of criminal prosecution. Vulnerability, considered broadly, consists of an individual being in a situation or condition in which she cannot adequately protect her own interests. The proposed guideline 15 for Council for International Organizations of Medical Sciences (CIOMS) states that researchers and research ethics committees must ensure that specific protections are in place to protect the rights and welfare of vulnerable groups enrolled in research. ${ }^{18}$

Understandably, concerns may be raised that in settings where PWID face oppressive practices, they should be viewed as a vulnerable population deserving special protection in (or from) research. When the research question addresses prevention or treatment of HIV among PWID, in most cases it will be necessary to enrol PWID in clinical research. However the question is whether choice of study sites or populations should be driven by selection of PWID who do not experience punitive laws, policies or practices, or who have better access to care, compared with those populations that do experience these oppressive conditions. It should be noted that these distinctions are all relative, since even in countries with more permissive laws and policies, social stigma and economic and cultural concerns can still be problematic. $^{22}$

Choice of study populations and research sites can affect the scientific validity and feasibility of a trial. Research in settings where HIV incidence is high is more likely to generate statistically meaningful results with respect to HIV-related outcome measures. Studies that are underpowered and unable to reliably answer the research question provide no scientific benefit. ${ }^{23}$ In terms of scientific feasibility, conducting appropriately designed 
HIV prevention studies in high-incidence populations has the greatest potential to answer the research question. Scientific feasibility is a necessary but not sufficient condition for research to go forward, since other ethical criteria must also be met.

Nevertheless, if the research question could easily be answered without enrolling PWID in oppressive settings, ethical guidelines for vulnerable populations would require specific justification for their inclusion. Such a default position is motivated by the goal of protecting vulnerable groups from exploitation or harm. In general, there are two types of justifications for inclusion of vulnerable groups: first, that provision of direct benefits of research is significant and outweighs risks, burdens or other adverse consequences of research participation; or, second, that developing effective policy change for these populations requires their participation in research studies, and that conducting the same research with different populations would fail to accomplish that goal. Both of these arguments come into play with regard to PWID. We believe direct benefits to trial participants must significantly outweigh risks for a trial to be ethically appropriate under difficult political conditions. Further, consideration of the likelihood of translation of research findings is also important, but may require special consideration (relative to other areas of clinical research) given the highly politicised nature of healthcare, harm reduction and other services for PWID. We discuss each of these issues in turn.

\section{Risks}

In addressing the ethical acceptability of research with PWID, it is critical to distinguish risks of research itself from the background risks experienced by PWID in the local setting. Both types of risk must be addressed by researchers in the design of the study and monitoring procedures.

\section{Addressing and minimising research risks}

As stated above, one formulation of the default position about protection of vulnerable groups is that it is unfair to expose people who already experience significant burdens from exclusion or oppression to additional risks associated with research itself. This default position is based on the assumption that research poses significant risks, or greater net risks than benefits. This is a rebuttable presumption, however, since some studies offer a favourable risk-benefit ratio and could provide direct benefits to disadvantaged groups, and at the same time produce valuable knowledge for future treatment or policy decisions relevant to their needs. The balance of risks to potential benefits varies across different studies. Phase I studies, for example, may be designed to expose participants to new products in order to assess safety, side effects, pharmacokinetics or establish dosing. The risks of phase I research vary greatly across studies, and can be significant. Usually the prospect of clinical benefit is unlikely. It is difficult to justify conducting a non-beneficial phase I study in a marginalised population already exposed to significant social and health burdens. In contrast to early phase research, some research on PWIDs seeks to quantify the relative therapeutic merits of interventions that are each likely to provide recipients with direct health benefit-providing potential benefit to all trial participants. Some commentators object to randomised trials with PWID, under the assumption that randomisation implies that risky experimental modalities are being used, that control groups will not benefit or that risks outweigh benefits. In fact, randomisation itself does not imply a high level of risk in these protocols, and each trial must be evaluated on the basis of risks and benefits of all trial arms.
In general, provision of direct benefits to trial participants is not sufficient in and of itself to justify research, since research must also pursue a scientifically valuable research question, but it is critically important to establish that benefits to PWID in clinical trials outweigh concerns about risks and burdens. In other words, given the significant burden of oppressive policies, lack of access to care and inadequate provision of harm reduction measures to PWID in many settings, it would be unreasonable to invite PWID to participate in studies with potential risk and no likelihood of direct benefit. With different populations, not similarly burdened it might be ethically acceptable to ask participants to make an altruistic contribution to research that is not directly beneficial. In sum, we advocate for ensuring direct benefits in HIV prevention studies involving high-risk PWID populations.

\section{Addressing and minimising background risks}

A related ethical challenge is that research may pose risks due to exacerbation of background risk for populations already facing stigma, violence, and legal and social disadvantages.

These issues were extensively examined in the context of the HIV Prevention Trials Network (HPTN) 058 study, which tested counselling plus buprenorphine substitution therapy for HIV prevention in China and Thailand. ${ }^{24}$ First, an intensive social and legal analysis of the setting of the research was conducted prior to initiating the trial. ${ }^{25}$ Second, site visits, community engagement and agreements with local authorities were secured prior to enrolment. Agreements with authorities were sought specifically to reduce the risk of incarceration or other adverse outcomes for study participants. Third, regular monitoring to ensure the social well-being of participants were part of routine study visits, by asking specifically about any difficulties experienced such as police/legal problems, housing, healthcare/insurance, employment, friend/family issues or other issues. The monitoring revealed that very little social harm occurred during participation in the trial: researchers documented a total of 4 incidents among the 1250 participants in the trial, all of which were described by the participants as minor: 3 had problems with friends and 1 had difficulty scheduling a study follow-up visit. $^{26}$ In fact, $80 \%$ of participants reported positive social impact of the trial at the 26-week visit, in such areas as reduction in drug use, better social relationships and economic improvement. It was also the case that some participants were incarcerated during the trial period, although there is no evidence that trial participation played any role, direct or indirect, in causing those events. Among those who were incarcerated, contact between researchers and authorities appeared helpful in securing their release, due to research contacts enabling families to advocate more effectively for their incarcerated family members, and most of the study participants were released. Experience from several trials, including HPTN $058^{24}$ indicates that with careful planning, research participation does not necessarily increase risk of adverse social or legal consequences, and in fact, could be protective. However it remains a concern that research has the potential to exacerbate risky background conditions, and it is a core ethical responsibility of researchers to carefully assess risks and take precautionary measures. If sufficient protections cannot be put into place, and if serious additional risks are likely to be triggered by the research itself, it would be unacceptable to initiate a trial in that setting.

\section{Distributive justice}

When studies provide significant benefits, selecting study populations whose rights are well protected while avoiding those who are experiencing rights violations, can disadvantage the latter 
group even further by setting back their interests in relation to their counterparts in less oppressive settings. Since HIV incidence is highest in settings where PWID lack access to appropriate care and legal protection, these groups need interventions more urgently than others in less risky settings. The ideal response to this need would involve a broad realignment of social policy and economic funding in order to make established effective interventions widely available in these settings. Our contention is that research that might alter restrictive social policy and that offers the prospect of direct benefit to participants may be permissible as a means of trying to ameliorate burdens experienced by PWID who live in environments that contribute to their susceptibility to disease and deprivation. In an environment with poor policies, evidence generated locally may have a much better chance of influencing future policieseven if the prospects for change are not bright-than evidence generated elsewhere. If there is some prospect of pushing productive policy change, we argue, it is better to include PWID from those settings in research than avoid them. This kind of claim must rest on evidence that there is at least a chance of progress, that chances will be made better by conducting research locally, and evidence that the research would not cause further harm, either directly or indirectly through adverse policy changes in response to the research activities.

Local efforts to improve care and services in the context of research, combined with new evidence regarding HIV risk, may have positive local effects. For example, researchers conducting an observational study near the northern Mexican border partnered with a local non-governmental organisation (NGO) using mobile vans for providing HIV prevention services for PWID. ${ }^{27}$ The researchers presented results of the study to local and regional health officials, demonstrating a worrisome increase in HIV incidence among PWID in the border region, and issued a joint binational press release. The study findings stimulated policymakers at the local and state levels in Mexico to adopt harmreduction approaches such as stepped-up provision of condoms and syringes. At the national level, a fleet of mobile vans was commissioned for SEP services.

Additional work is often needed to extend and sustain improvements in clinical care at the conclusion of a research study. It is difficult to secure guarantees of sustained care in the context of research budgets, but partnership with local health authorities, where possible, as well as involvement of NGOs and community groups, may improve sustainability and continuity in some cases. And in fact, there is some evidence that policymakers look preferentially to locally generated evidence when making decisions about implementation at the local level. ${ }^{28}$

At a broader level, advocacy groups, expert bodies and NGOs gather and present evidence from research to advocate internationally for better policies and programmes for PWID and to inform international guidelines. WHO guidance can prompt health policy changes, and current WHO guidelines call for the use of a series of interventions for PWID that address healthcare needs and HIV risk: community-based outreach, SEPs, OST and other drug-dependence treatment; HIV counselling and testing; ART for injection drug users living with HIV; prevention and treatment of sexually transmitted infections; condom programmes for IDUs and their sexual partners; targeted information, education and communication for IDUs and their sexual partners; vaccination, diagnosis and treatment of viral hepatitis; and prevention, diagnosis and treatment of tuberculosis. ${ }^{5}$ These guidelines are intended to drive positive policy change, and in spite of the challenges of oppressive policies in many countries, there are some success stories. For example, OST is now offered to PWID in Mauritius and Tanzania and is being implemented in Kenya. $^{29}$ If research was avoided systematically in settings with problematic policy developments due to concerns about uneven implementation, the status quo would prevail, and the interests of these disadvantaged groups would continue to be underaddressed, contributing to ongoing and worsening inequalities in care, and exposure to HIV risk.

\section{RESPONSIVENESS AND REASONABLE AVAILABILITY REQUIREMENTS: WILL RESEARCH FINDINGS BE TRANSLATED INTO HEALTH PRACTICE?}

International ethics guidelines regarding research in resourcelimited settings emphasise the need for responsiveness to host country priorities: research studies should address a priority health need and have the potential to provide benefit to those in the host country. ${ }^{18} 19$ The concern is that some research studies may exploit those living in poor countries by testing products or approaches that are irrelevant to health needs of the host country and that in the end will only benefit other, wealthier countries or regions. A related concern is reasonable availability of interventions that are proven effective. ${ }^{4}$ There is often a major gap in delivery of proven interventions in resource-poor settings, and if barriers to implementation are not addressed, study findings, again, may benefit only wealthier regions and countries that host clinical trials will not reap rewards of improvement of health for their populations. ${ }^{30}$ Thus, questions arise as to whether clinical trial findings will influence policy in settings where legal and political forces are major obstacles for treatment and care for PWID. ${ }^{4}$

In the bioethics literature, debates about responsiveness and reasonable availability have typically centred on situations where resource constraints are the principal challenge to effective implementation of new interventions. However, in research with PWID, research may be ignored by governments that employ non-evidence-based policies driven by political considerations rather than public health objectives. In the case of harm reduction for PWID, then, the barriers typically relate more to political concerns than cost.

Moreover, policy decisions regarding proven, evidence-based interventions for harm reduction can be unpredictable and idiosyncratic. Ukraine has implemented a policy providing OST to PWID in recent years, although access and availability of services is not yet universal, whereas in Russia, OST remains illegal. With the recent takeover of the Crimean peninsula by Russian forces, OST was abruptly suspended for approximately 600 opiate-addicted persons. ${ }^{31}$ In the USA, a congressional ban on US federal funds to support SEPs was in effect until 2012, briefly lifted and then reinstated in 2013, which has led to significant difficulties in sustaining SEPs in high-risk metropolitan areas where most PWIDs reside. The ban on federal funding for SEPS was lifted yet again in December $2015,{ }^{32}$ reflecting the everchanging political and legislative climate with regard to this public health intervention. The unpredictability of these political forces raises questions about whether an improved evidence base can make a positive contribution to changes in policy and practice. Recently, an HIV outbreak in Indiana ${ }^{33} 34$ has prompted lawmakers in that and other states to reconsider SEPs. While the introduction of new SEPs is encouraging, these developmentsthe timing of the outbreak itself, and the response of local authorities-were not predictable in advance and do not provide a reliable framework for linking research projects with policy action.

In situations where politics trump science in decision-making, and where effective interventions for PWID are not available, 
questions arise about the ethical acceptability of research trials. Should researchers avoid engaging in settings where policymaking processes do not appear responsive to the evidence base?

The relationship between evidence and policymaking is, in fact, complicated and unpredictable even in less contentious settings. ${ }^{35}$ Several models of policymaking have been described and each has implications for non-evidentiary concerns playing a major role, for example, the influence of established positions within organisations, casual empiricism and speculation, policy networks of various actors, and political processes. ${ }^{35}$ Models of research utilisation also include a variety of processes such as strategic or adversarial politics, social and professional interactions, and the institutionalisation of knowledge. ${ }^{36-38}$ What all these models have in common is a combination of various actors and political and social conditions that are needed to accomplish policy change. In the public health arena, researchers certainly constitute one important stakeholder group, but are far from being the major or decisive factor in policy decisions. In fact, some analysts describe difficulties in achieving policy change regarding drug use as being due in part to the divergent views of public health and criminal justice authorities:

Several explanations emerged to explain this phenomenon. The first is that drug policy making remains opaque and divided between the more powerful law enforcement and justice ministries that support punitive approaches, and the health ministries which tend more toward supporting harm reduction approaches to managing drug use. ${ }^{39}$

Recently, public health researchers described a series of case studies regarding policymaking on SEPs in three US cities. ${ }^{40}$ In one of these cities, Baltimore, the relationships between researchers and policymakers seemed to play a positive role in supporting evidence-based policy. In contrast, in Philadelphia, grassroots organising, following the evidence base was critical; and in Washington DC, political change was the major factor. In the face of diverse policy responses, researchers usually cannot predict when or how findings will be implemented in public health programmes. Certainly many researchers become advocates for better evidence-based approaches; ${ }^{34}$ and many public health researchers are also champions for human rights of PWID in international policy discussions. ${ }^{12}$ It is unclear when and how these activities might bear fruit in terms of real implementation of productive programmes for PWID, in spite of substantial effort on the part of the research community in advocating for policy change. In any of these efforts for policy change, it is clear that partnership and collaboration with community representatives from PWID organisations and advocacy groups is essential. These partnerships help ensure that policy change will be more responsive to the best interests and health needs of PWID.

Focus on the responsiveness question in the bioethics literature has led some to conclude that research is simply not justified in settings where research findings cannot be put into practice, because any risks or burdens of research would be unfairly imposed on individuals and communities who cannot later reap the benefits of successful interventions. ${ }^{41}$ Proponents of this ethical requirement have discussed the difficulty in determining precisely which research projects satisfy the responsiveness and reasonable availability requirements, how much flexibility should be allowed, and what procedural mechanisms could be used to make these judgments. The difficult policy environment regarding healthcare and services for PWID only serves to make this analysis more challenging.

In spite of the complexity of the responsiveness and reasonable availability principles and lack of consensus regarding their application, there are three main arguments in favour of including PWID in studies from specific countries where policy processes are uncertain. The first relates to possibilities of local, national or regional change. Although some policymakers may not be motivated solely by a desire to protect the rights and interests of PWID, it may be possible to persuade them that HIV prevention policies protecting PWID are worthwhile if they are cost-effective at the population level. For example, in many locations, police education programmes that bundle occupational safety with harm reduction messages have been met favourably by police departments ${ }^{16} 22{ }^{42}$ which offers the possibility that police may be convinced to avoid behaviours that elevate HIV risks among PWID. Moreover, policymakers may be resistant to implement programmes on the basis of data generated in social contexts that are very different from their own. Trials that generate evidence regarding local efficacy and cost-effectiveness of interventions for PWID may therefore meet the requirement of responsiveness to local health needs. ${ }^{18} 19$ Positive policy developments have occurred, for example increasing numbers of PWID receiving OST in China and Vietnam, and increases in ART coverage for PWID in Vietnam. Ukraine has significantly increased SEP coverage, and Malaysia has made a dramatic shift from a punitive law enforcement approach to evidence-based prevention and treatment programmes. ${ }^{43}$

The second argument relates to the potential for direct benefit to trial participants. Although the overall purpose of studies is to advance the knowledge base for prevention, care and treatment interventions for PWID, many clinical trials in this area offer a favourable balance of risks and benefits to participants, as when studies compare the relative merits of prevention modalities that are known to be effective. In such cases, participation in research may represent the only avenue through which PWID can access prevention modalities that are restricted on the basis of law or policy. When this is the case, excluding certain populations of PWID from the direct benefits of research may exacerbate their health burdens and also constitute unfairness. Decisions about whether the overall benefits of participating in a research study outweigh other ethical concerns must include the voices and opinions of PWID and their representatives in each local setting. Community engagement has become standard practice in HIV prevention research, but the degree of input and control exerted by community representatives and advisory boards varies substantially across studies and contexts. True representation of PWID in evaluating the acceptability of clinical research studies is critically important given the array of social and ethical issues at stake. ${ }^{44}$

The third argument for not requiring a predictable path towards policy change is that when political conditions are favourable for change, evidence must already be in hand. Waiting for political forces to come into alignment to then generate new research studies would often be too late. Clinical trials take years to implement and generate findings; and if a political favourable environment emerged without the requisite evidence being available, valuable opportunities would be lost. Policymakers might choose to wait for evidence to be generated (which would take time, and further delay positive impacts for PWID populations); or they might act without having reliable evidence in handwhich could lead to non-evidence-based or ineffective approaches being implemented.

\section{STANDARDS OF CARE AND PREVENTION: WHAT STANDARD OF CARE AND PREVENTION IS ETHICALLY APPROPRIATE?}

In designing research, it is essential to determine the appropriate standard of care and standard of prevention. In the context of 
HIV prevention trials, standard of prevention often refers to the set of services that will be provided to all participants in the proposed research regardless of the study arm, although this could also refer to active control arms in clinical trials. Standards of care and prevention affect several important ethical domains of the research, including protection of well-being of trial participants, scientific integrity of the research, utility of trial results for policy decision-making, and ultimately, fairness in distribution of resources to address health needs in populations. While standard of prevention questions arise in almost all HIV prevention research, research with PWID must grapple with the additional legal, political and social complexities of drug use, and the substantial barriers at multiple political and operational levels to implementation of known prevention tools.

The Bangkok Tenofovir Study, for example, demonstrated the safety and efficacy of tenofovir for pre-exposure prophylaxis among PWID ${ }^{45}$ but the study has been criticised in part because an evidence-based means of prevention (ie, SEP) was not used either as background care or as the comparator arm of the trial and study treatment was made available in closed settings even though this would not be expected outside of the research setting. $^{46}$ Some local advocacy groups representing PWID opposed the trial due to the lack of provision of clean syringes in the trial, and the lack of significant input into the study protocol. $^{47}$ Concerns were expressed that recruitment was carried out by nurses who delivered methadone treatment, compromising voluntariness of participation. Advocates also reported that only certain individuals were invited to participate in community advisory board discussions. The debates about the Bangkok trial demonstrate the importance of addressing broad community representation and ensuring engagement early in the research process so that advocates and representatives have a voice in the design of the trial. ${ }^{48}$ Input from stakeholders on standards of care and prevention is critical as the trial is being designed, as the data are collected and as the results are disseminated. ${ }^{44}$

In the context of clinical trials, there have been vigorous debates about how to handle situations where the healthcare system delivers a low level of care, that is, when the local 'standard' does not reflect accepted evidence-based practices.

A fundamental tension relates to whether it is appropriate to use the local standard of care as the prevention standard with the aim of maximising local relevance and feasibility, versus implementing higher standards of care or prevention with the goal of driving or pushing standards to a higher level and providing highest level of direct benefit to study participants. There are important trade-offs in terms of the presumed benefits of higher standards versus the presumed greater feasibility of implementing local standards in the healthcare system. Substantial bioethics debate on this topic took place in the context of trials of antiretroviral interventions to prevent mother-to-child transmission of HIV. ${ }^{49-51}$

International guidance documents also differ on what the standard of prevention ought to entail. For example, the Declaration of Helsinki exhorts researchers to use the best known effective clinical standards in a clinical trial. In contrast, the CIOMS guidelines allow for using lesser than best standards due to considerations of local context, provided there is specific justification for the standard chosen.

The UNAIDS Ethical Considerations in Biomedical HIV Prevention Trials, Guidance Point 13 states that "all state of the art risk reduction methods" should be provided to participants in clinical trials "as they are scientifically validated or as they approved by relevant authorities," whether or not these interventions are available locally. ${ }^{52}$ The guidance also specifically addresses research with PWID, and states that "where there are insurmountable barriers to ensuing access to sterile needles and syringes for all trial participants, HIV prevention trials among people who inject drugs should not proceed." This guidance point raises particularly challenging issues for efficacy trials on HIV prevention with PWID, since many countries with a high prevalence of PWID and HIV infection have outlawed SEPs and OSTs.

Finally, the HPTN Ethics Guidance takes a pragmatic approach, advocating for locally sustainable standards to be used in trials. ${ }^{53}$ For a particular method to be included in the standard of prevention there must be evidence of efficacy, it must be reasonably accessible and be practically achievable in the research setting. The rationale for this approach is that sustainable standards address considerations of locally distributive justice, as well as ensure that trials are relevant to the local setting.

This wide variation in ethical guidance on the topic of standards of care and prevention highlights the difficulty in reaching a strong consensus on an ethical path forward. Arranging the various options on a continuum, the least demanding standard is using the current local standard of care in a trial, while the most demanding regime would be to provide all known efficacious measures to study participants. Arguments for simply accepting the status quo standards are easy to defeat, since the ultimate objective of clinical research is to improve standards of care, both in terms of organised delivery of known interventions as well as testing new interventions or strategies, and local care may simply be well below any acceptable level. Arguments in favour of de facto standards are often based on the idea that the local standard has maximum relevance to local conditions, coupled with the claim that researchers do not have specific obligations to trial participants to remedy conditions of healthcare disparity that they did not create. However, aiming for relevance should not lead to absolute judgments on accepting the status quo. ${ }^{54-56}$

On the other end of the spectrum, an argument in favour of delivering all known interventions, as advocated by UNAIDS guidance $^{52}$ may be criticised on the basis that it is unrealistic that health systems are going to simultaneously adopt all proven methods, even if political obstacles were removed. Cost-effectiveness and priority setting in health systems inevitably results in some difficult decisions about what services will be funded. Moreover, employing all methods may be logistically challenging, or potentially cause unintended clinical or behavioural side effects related to the interactions of methods that have not been previously combined. While the UNAIDS guidance is international and intended to apply broadly to HIV research around the world, in fact, nuances in its application have been discussed in several commentaries ${ }^{56} 57$ and stakeholders have expressed various views of the feasibility of implementing all its provisions on the ground. ${ }^{58}$ For example, when local regulatory authorities have not approved an intervention, in some cases it may be practically impossible to deliver it as a background intervention in a trial. And in spite of the international standing of this guidance, there is wide variability in how standards of prevention are interpreted in practice, depending on the design of the trial, needs of the population and local healthcare capacity. $^{59}$

The HPTN ethics guidance advocates feasible and sustainable improvements to standards of care and prevention, which provides a middle ground in establishing better standards of prevention when local clinical standards are so low as to allow significant and preventable harm ${ }^{53}$ Improvements in the standard of care defined by these criteria help protect welfare of trial participants, retain relevance to local healthcare systems and help 
drive improvements in clinical standards. In spite of the theoretical appeal of this argument, the question that arises is how high a level of improvement can be implemented and sustained, especially in light of political and legal complexities relevant to interventions for PWID.

Choice of the standard of prevention also affects scientific aspects of clinical trials and trial efficiency. ${ }^{56}$ In prevention trials that use HIV as an end point, the more infections that are prevented across all study arms, the greater the sample size needed to detect the effect of the intervention being studied. Similarly, the more interventions that are included in a trial, the more complex it may be to detect the effect of any single intervention. ${ }^{23} 56$

Clinical trials have the potential to provide both an evidence base and pragmatic experience with better care and services for PWID. Research trials sometimes provide a platform to test treatment modalities that are politically unpopular, thus gathering evidence that can be used in policy and advocacy discussions to improve care and treatment. However, in some settings it is impossible to introduce specific prevention or treatment modalities into a trial when government authorities refuse to approve the trial or have banned the interventions.

A particularly controversial aspect of the standard of prevention arises when clinical trials are conducted in countries that do not have SEP. If provision of syringes is outlawed in certain settings, researchers do not have the option to offer this service as part of the trial. The question is whether researchers should be allowed to include sites without SEP programmes, whether they should make arrangements to provide services even when not locally available or whether those sites and countries should be excluded from research entirely.

Advocates for rights of PWID around the globe, including many in the research and public health communities as well as diverse and numerous NGOs ${ }^{60}{ }^{61}$ have been consistently calling for improved access to harm reduction interventions such as SEP and OST. ${ }^{4}$ These interventions are proven effective and costeffective in reducing the risk of acquiring HIV and viral hepatitis and can help engage PWID in clinical care. There is a risk that biomedical trials for HIV prevention being conducted in anything other than a setting with full access to SEP and other services for PWID will be viewed as support for these negative policies, and might encourage 'medicalisation' of HIV prevention. These developments would not serve the interests of PWID and could derail the emphasis on essential services that should be provided for all PWID but are often politically unpopular. Some commentators express concern that even the existence of trials of other strategies for HIV prevention provide de facto support for bad policies denying SEP and OST to those who need them and distract from the push for progressive policy change. ${ }^{4}$ Another objection is that use of antiretroviral (ARV)-related approaches like pre-exposure prophylaxis (PrEP) pose an extra burden on PWID-pill taking, side effects, challenges in maintaining drug regimens-for objectives related to population health and not primarily for individual benefit.

Analysing these objections requires further exploration of the important claims at stake. There is strong consensus among international health organisations that SEP and OST ought to be standard interventions available to all people who need them, and ARV-based interventions would not be as urgently needed if clean needles were available. By analogy, if a hospital failed to follow proper infection control procedures, all its patients might need to be treated with antibiotics either prophylactically or therapeutically. But if standard infection control were observed, the treatment would not be necessary. A call for appropriate hospital procedures would be in order-just as the call for SEP is made by advocates and NGOs and researchers around the world. We suggest that all HIV prevention researchers, including those conducting biomedical prevention trials, join this call to action to ensure that the minimum package of nine HIV prevention interventions for PWID $^{5}$ is available in all settings where they propose to conduct research.

On the other hand, there are times when structural change takes time, and significant political barriers to SEP and OST have been very difficult to address. A different analogy could be found in early arguments for the development of femalecontrolled methods for HIV prevention-at a time when the only intervention was the male condom, which many men refused to use. Women's rights advocates continued to press for better legal and social protection systems for women so that they are not forced into sexual relations without protection from HIV. At the same time, advocates for further HIV prevention research wanted to explore female-controlled methods like vaginal microbicides that would give women an option to choose to protect themselves even when their partners didn't or wouldn't cooperate. Research advocates also supported the agenda for women's rights, but wanted to look for mechanisms to protect women in the near term-without waiting for the massive cultural, political and legal changes needed to guarantee women full decisionmaking autonomy.

The situation with SEP and PWID resembles each of these scenarios to some extent. It is unconscionable that known safe, affordable and effective public health interventions like SEP and OST continue to be rejected for overtly political reasons in many settings. Basic human rights and public health principles should dictate that these ill-conceived, non-evidence-based policy choices be reversed. At the same time, PWID deserve to have access to other beneficial interventions as well. In a fair world, PWID everywhere have access to the entire array of interventions -SEP, OST and use of ARVs, for prevention and treatment. Community advocates and researchers recognise that an array of prevention methods is needed in each setting so that individuals can choose the particular combination that is feasible and acceptable to them.

The development of new evidence for prevention treatment and care should not be a substitute for advocacy for policy and legal change. And many researchers and advocates around the world believe that researchers should set an example of proactive approaches to higher standards of prevention in clinical trials. Specifically, advocates have called for provision of sterile injecting equipment to be a uniform requirement for any trials with PWID. ${ }^{62}$ We endorse this requirement as the default position, and a principled approach to the conduct of clinical trials. Any deviation from this approach could only be undertaken with strong endorsement from locally representative advocacy groups for PWID, and only after satisfying the other ethical requirements we have laid out: favourable risk-benefit ratio, community engagement, and steps taken, as far as possible, to promote uptake of successful research interventions. In other words, any deviation from globally accepted standards of prevention for PWID could only be undertaken if a study offered direct benefits and was endorsed by the local community as ethical and appropriate to meet their needs.

Some important trials have used locally relevant standards and have developed new, effective and feasible interventions. For example, despite stringent policies towards PWID and no access to methadone or buprenorphine maintenance for PWID in Russia, research showed that naltrexone (an opioid receptor antagonist) can be used effectively to manage opioid 
dependence $^{63}$ and that a peer educator network approach is a promising strategy to reducing HIV incidence. ${ }^{64}$ This example illustrates that in some cases, despite government prohibitions on use of specific effective interventions, alternative interventions may be tested and may provide much needed benefits to PWID.

In working to determine the appropriate standard of prevention for a HIV-related research with PWID, researchers and sponsors can address these social, cultural, ethical and political tensions in several ways. First, investigators should consult meaningfully and broadly with community representatives and advocates for PWID in the local setting and receive input from these groups about clinical trial design, procedures and dissemination of the research. ${ }^{44}$ Researchers should also work with local colleagues to assess conditions on the ground-such as the availability of interventions, and potential cultural or political resistance to implementation. Investigators should also seek to form alliances with local key groups, including PWID organisations, health organisations, patient organisations, NGOs and government representatives. Researchers can interact closely with these stakeholders to assess the local policy environment, including the status of criminal and public health laws and policies pertaining to drug use and prevention-what these laws are, whether they are implemented, and if so, when and how effectively. Researchers should also collaborate with local colleagues to understand the standard of care, and the standard of prevention, and research needs. Investigators should develop a research strategy with the goal of providing the best possible set of interventions that could be made available. This list of interventions may exceed what is currently available, but should constitute a realistic horizon. Discussions with stakeholders can also address the questions raised above concerning whether researchers will be seen as being complicit with bad governmental policy, or whether pressure on policymakers will eventually prompt delivery of better care.

\section{Scientific viability}

There can be trade-offs in optimising higher standards of care versus optimising efficiency when choosing prevention methods for clinical trials. ${ }^{56}$ In terms of efficiency, there is generally no bright line between 'possible' and 'impossible' clinical trials, but rather, a gradation of trial sizes and costs. Responsible use of resources for public good is an ethically important objective, and therefore efficiency is also an ethical concern. For a clinical trial to be a viable project, minimum standards for scientific validity and integrity must be met. There is no single appropriate answer to balancing trial efficiency concerns with other ethical criteria; however, there are standards for protection of welfare and safety of trial participants that must be met in all cases.

\section{COMPLICITY: IS IT ETHICALLY PERMISSIBLE TO DO RESEARCH IN SETTINGS WITH OPPRESSIVE POLICIES?}

Another concern about research in oppressive settings is that the presence of researchers and the substantial flow of research funding needed to conduct clinical trials, may inadvertently support negative policies and practice, or give economic or practical support to agencies with oppressive policies. At a more abstract level, there is a concern that researchers' activities can 'send a message' that these oppressive policies are acceptable. The question is whether researchers or sponsors should impose a boycott or moratorium on research in settings where basic rights and services for PWID are not protected by local laws and policies, even when research participation offers direct tangible benefits to PWID. As a minimum standard, researchers should provide improved services for PWID in the trial, protect confidentiality and ensure that study participation does not engender social harm-but there remain questions about whether researchers' engagement could somehow signal support or collusion with oppressive policies.

By way of analogy, consider the use of trade sanctions against regimes that violate human rights or have other morally unacceptable policies affecting their own populations. Sometimes opponents of trade sanctions argue that they are ineffective at changing policy and that they only serve to cause greater economic hardship for vulnerable members of the target country. Proponents of sanctions argue that 'putting the squeeze' on governments with reprehensible policies will ultimately force them to change, and furthermore, they might see this as a principled stand: countries that impose sanctions are forgoing the benefit they might obtain through active trade agreements, with the aim of forcing policy change in the target country. This analogy, however, does not fit the scenario for research on stigmatised groups like PWID. In contrast to trade sanctions, a boycott or moratorium on research projects in countries that stigmatise or criminalise PWID will not harm the interests of the government or the majority of the population-if anything, it has the potential to derail advancement of the interests of PWID who arguably can stand to benefit both directly and in the longer term, from research. Even in the case where it is uncertain that long-term benefit (of policy change) will emerge, but the balance of risks and benefit to study participants is reasonable, a decision not to conduct the research will not advance the interests of PWID. The pursuit of research on prevention of HIV and related healthcare for PWID is unlikely to be politically popular, and a failure to initiate these kinds of research projects will not create any hardship for policymakers, or exert real political pressure to drive policy changes.

Even if it is clear that boycotting specific countries will not have concrete impact on policies, some commentators believe that situating research in a country with oppressive practices on some level sends a message of approval of the host country's approach. There is no documented evidence from previous studies that host countries perceive researchers this way and so this question remains open. In fact, researchers are among the strongest advocates for constructive change in regard to health policies for PWID and other vulnerable groups. It is appropriate for researchers to use their specialised expertise and specific study findings to advocate for better public health approaches for PWID, whenever possible.

\section{CONCLUSION: CRITERIA FOR ETHICALLY ACCEPTABLE RESEARCH WITH PWID IN OPPRESSIVE SETTINGS}

Based on our analysis, we propose a set of basic criteria for determining ethical acceptability of proposed research with PWID that supplement conventional scientific and ethical standards for research. The criteria are inter-related, in that satisfaction of one criterion may depend on resolution of the others-but each represents a distinct ethical concern and all the criteria must be met to ensure that adequate ethical protections are in place.

A. The study must fill an important evidence gap which could be used to advance policy to improve healthcare or services for PWID in one or more host countries, even if the need for policy change makes practice implementation on a broader basis uncertain in the near term;

B. Community consultation with PWID and relevant advocacy organisations for PWID should take place before the trial and should inform study design, procedures and dissemination. Community input should not be relegated to reviewing consent documents or 'rubber stamp' of protocols but should 
include substantive discussions about the relevance and acceptability of the research plan and execution;

C. Welfare of study participants should be carefully addressed through risk mitigation procedures such that the research does not exacerbate underlying conditions of criminalisation, stigma and violence;

D. Clinical trials that involve greater than minimal risk should provide an offsetting prospect of direct benefit to trial participants, and potential risks of research interventions should be counterbalanced by direct benefits;

E. The standard of prevention must be established at the highest standard that can be feasibly and safely delivered at the research sites; provision of sterile injecting equipment must be seen as a basic ethical requirement in all studies;

F. Research teams should contribute to advancing policy discussions in host countries whenever possible, including collaborating with local advocacy and community-based organisations for PWID; and

G. Researchers should support and contribute to global advocacy for evidence-based standards of care and treatment and human rights protections for PWID.

At the extreme, there are cases in which evidence-based HIV prevention methods are illegal in host countries, calling into question the viability of structuring clinical trials with active control arms or prevention packages. However, even in these settings, clinical trials can make positive contributions to evidence and policy development, assuming these six criteria are satisfied and the research is designed and conducted according to accepted scientific and ethical standards.

It is also important to define conditions under which research should not be conducted. Studies that do not offer clinical benefit and involve greater than minimal risk should not be undertaken in populations of PWID who are already disadvantaged by legal, social and political structures. Studies that will predictably increase the likelihood or severity of harm due to exacerbation of background conditions should likewise not go forward. The aim, therefore, should be for research sponsors and researchers to support and conduct research that addresses important evidence gaps, protects PWID from increases in background risk and provides some clinical benefit to PWID, while making the best possible effort to impel constructive policy changes, although in an uncertain environment.

Whether trials should go forward under adverse political and social conditions involves balancing risks and benefits to trial participants, determining whether the trial can serve to improve conditions or policies in the host country, taking efforts to minimise the likelihood that the trial would serve as either implicit or explicit endorsement or support of practices that deny rights and services to PWID, and considering the risks or costs of failing to do research on important health questions for PWID. We hope that discussion and deliberation of our proposed criteria will help focus discussion about the appropriateness of particular research initiatives and contribute to refinement of policy in this regard.

Further scholarship and policy work needs to be directed at how the research community can constructively engage with policymakers to advance the health agenda for PWID, in addition to conducting high quality research to address evidence gaps.

Contributors All authors contributed to conceptual analysis and drafting of the manuscript. All authors approved the final version of the manuscript. The views expressed in the article are those of the authors alone and do not represent the views or policies of the US National Institutes of Health or any of its components.

Funding US Department of Health and Human Services, National Institutes of Health (UM1AI068619).
Competing interests None declared.

Provenance and peer review Commissioned; externally peer reviewed.

\section{REFERENCES}

1 Joint United Nations Programme on HIVIAIDS. Global Report: UNAIDS report on the global AIDS epidemic. 2013.

2 Strathdee SA, Stockman JK. Epidemiology of HIV among injecting and non-injecting drug users: current trends and implications for interventions. Curr HIVIAIDS Rep 2010;7:99-106.

3 Mathers BM, Degenhardt L, Ali $\mathrm{H}$, et al. HIV prevention, treatment, and care services for people who inject drugs: a systematic review of global, regional, and national coverage. Lancet 2010;375:1014-28.

4 International Network of People who Use Drugs (INPUD). An Introduction to Pre-Exposure Prophylaxis (PrEP) for People who Inject Drugs: pros, cons and concerns. http://www.inpud.net/en/background-document-introduction-pre-exposureprophylaxis-prep-people-who-inject-drugs

5 World Health Organization, United Nations Office on Drugs Crime, Joint United Nations Programme on HIVIAIDS. Technical guide for countries to set targets for Universal Access to HIV prevention, treatment and care for injecting drug users. Geneva, Switzerland: WHO, UNODC, and UNAIDS, 2009. http://apps.who.int/iris/ bitstream/10665/77969/1/9789241504379_eng.pdf

6 Strathdee SA, Shoptaw S, Quan V, et al. for the substance use scientific committee of the HIV prevention trials network. Towards combination HIV prevention for injection drug users: addressing addictophobia, apathy and inattention. Curr Opin HIV AIDS 2012;7:320-5.

7 Petersen Z, Myers B, van Hout MC, et al. Availability of HIV prevention and treatment services for people who inject drugs: findings from 21 countries. Harm Reduct J 2013;10:13

8 Wood E, Kerr T, Tyndall MW, et al. A review of barriers and facilitators of HIV treatment among injection drug users. AIDS 2008;22:1247-56.

9 International Harm Reduction Association. Inflicting harm: Judicial corporal punishment for drug and alcohol offencesin selected countries. Secondary Inflicting harm: Judicial corporal punishment for drug and alcohol offencesin selected countries. 2011. http://www.ihra.net/files/2011/11/08/IHRA_CorporalPunishmentReport_Web.pdf (accessed May 2015).

10 Shaboltas AV, Skochilov RV, Brown LB, et al. The feasibility of an intensive case management program for injection drug users on antiretroviral therapy in St. Petersburg, Russia. Harm Reduct J 2013;10:15.

11 Degenhardt L, Mathers B, Vickerman P, et al. Prevention of HIV infection for people who inject drugs: why individual, structural, and combination approaches are needed. Lancet 2010;376:285-301.

12 Wolfe D, Cohen J. Human rights and HIV prevention, treatment, and care for people who inject drugs: key principles and research needs. J Acquir Immune Defic Syndr 2010;55(Suppl 1):S56-62.

13 Strathdee SA, Beletsky L, Kerr T. HIV, drugs and the legal environment. Int I Drug Policy 2015;26(Suppl 1):S27-32.

14 Hayashi K, Ti L, Csete J, et al. Reports of police beating and associated harms among people who inject drugs in Bangkok, Thailand: a serial cross-sectional study. BMC Public Health 2013;13:1-8.

15 Booth RE, Dvoryak S, Sung-Joon M, et al. Law enforcement practices associated with HIV infection among injection drug users in Odessa, Ukraine. AIDS Behav 2013;17:2604-14.

16 Beletsky L, Agrawal A, Moreau B, et al. Police training to align law enforcement and HIV prevention: preliminary evidence from the field. Am J Public Health 2011:101:2012-15.

17 Mimiaga MJ, Safren SA, Dvoryak S, et al. "We fear the police, and the police fear us": structural and individual barriers and facilitators to HIV medication adherence among injection drug users in Kiev, Ukraine. AIDS Care 2010;22:1305-13.

18 The Council for International Organizations of Medical Sciences (CIOMS). International ethical guidelines for biomedical research involving human subjects. Bull Med Ethics 2002:17-23.

19 World Medical Association. Declaration of Helsinki, ethical principles for medical research involving human subjects. 52nd WMA General Assembly. Edinburgh, Scotland. 2000.

20 Levine C, Faden R, Grady C, et al. The limitations of "vulnerability" as a protection for human research participants. Am J Bioeth 2004;4:44-9.

21 DuBois JM, Beskow L, Campbell J, et al. Restoring balance: a consensus statement on the protection of vulnerable research participants. Am J Public Health 2012;102:2220-5.

22 Park JN, White $B$, Bates $A$, et al. Motivators and barriers influencing willingness to participate in candidate HCV vaccine trials: perspectives of people who inject drugs. Drug Alcohol Depend 2012;123:35-40.

23 Donnell D, Hughes JP, Fleming TR. Challenges in the design of HIV prevention trials in the United States. J Acquir Immune Defic Syndr 2010;55(Suppl 2):S136-40.

24 Metzger DS, Donnell D, Celentano DD, et al. HPTN 058 Protocol Team. Expanding substance use treatment options for HIV prevention with Buprenorphine-Naloxone: HIV prevention trials network 058. J Acquir Immune Defic Synr 2015;68:554-61. 
25 Youchun F, Wang D. HPTN 058: Rapid Policy Assessment for China and Thailand: Family Health International. 2007.

26 Sugarman J, Rose SM, Metzger D. Ethical issues in HIV prevention research with people who inject drugs. Clincal Trials 2014;11:239-45.

27 Strathdee SA, Magis-Rodriguez C, Mays VM, et al. The emerging HIV epidemic on the Mexico-U.S. border: an international case study characterizing the role of epidemiology in surveillance and response. Ann Epidemiol 2012;22:426-38.

28 Oliver K, de Vocht F. Defining evidence: a survey of public health policy makers needs and preferences. Eur J Public Health 2013:http://dx.doi.org/10.1093/eurpub/ ckv082.

29 Lambdin BH, Masao F, Chang O, et al. Methadone treatment for HIV preventionfeasibility, retention, and predictors of attrition in Dar es Salaam, Tanzania: a retrospective cohort study. Clin Infect Dis 2014;59:735-42.

30 London AJ. Responsiveness to host community health needs. In: Emanuel EJ, Crouch R, Grady C, et al. eds. The Oxford textbook of clinical research ethics. New Yok, Oxford University Press, 2008:737-744.

31 Kazatchkine M. Russia's ban on methadone for drug users in Crimea will worsen the HIV/AIDS epidemic and risk public health. BMJ 2014;348:93118.

32 Ungar L. Funding ban on needle exchanges effectively lifted. USAToday, 7 January 2016. http://www.usatoday.com/story/news/nation/2016/01/07/funding-ban-needleexchanges-effectively-lifted/78420894/

33 Conrad C, Bradley HM, Broz D, Community outbreak of HIV infection linked to injection drug use of oxymorphone - Indiana, 2015. MMWR Morb Mortal Wkly Rep 2015:64:443-4.

34 Strathdee SA, Beyrer C. Threading the needle- - how to stop the HIV outbreak in rural Indiana. threading the needle- how to stop the HIV outbreak in rural Indiana. N Engl J Med 2015;373:397-9.

35 Hanney SR, Gonzalez-Block MA, Buxton MJ, et al. The utilization of health research in policy-making: concepts, examples and methods of assessment. Health Res Policy Syst 2003;1:2.

36 Bowen S, Zwi AB. Pathways to "evidence-informed" policy and practice: a framework for action. PLoS Med 2005:2:e166.

37 Brownson RC, Royer C, Reid E, et al. Researchers and policymakers: travelers in parallel universes. Am J Prev Med 2006;30:164-72.

38 Field P, Gauld R, Lawrence M. Evidence-informed health policy-the crucial role of advocacy. Int J of Clin Pract 2012;66:337-41.

39 "Drug policy advocacy in Asia: Challenges, opportunities and prospects," International Drug Policy Consortium: http://www.aidsdatahub.org/sites/default/files/ publication/drug_policy_advocacy_in_asia_2013.pdf

40 Allen ST, Ruiz MS, O'Rourke A. The evidence does not speak for itself: the role of research evidence in shaping policy change for the implementation of publicly funded syringe exchange programs in three US cities. Int I Drug Policy 2015;26:688-95.

41 Benatar SR. Towards progress in resolving dilemmas in international research ethics. J Law Med Ethics 2004;32:574-82.

42 Beletsky L, Thomas R, Shumskaya N, et al. Police education as a component of national HIV response: lessons from Kyrgyzstan. Drug Alcohol Depend 2013;132 (Suppl 1):S48-52.

43 Degenhardt L, Mathers BM, Wirtz AL, et al. What has been achieved in HIV prevention, treatment and care for people who inject drugs, 2010-2012? A review of the six highest burden countries. Int I Drug Policy 2014;25:53-60.

44 Jurgens R. Nothing about us without us - Greater, meaningful involvement of people who illegal drugs: a public health, ethical and human rights imperative. International edition. Toronto: Canadian HIV/AIDS Legal Network, International HIVIAIDS Alliance, Open Society Institute. 2008.

45 Choopanya K, Martin M, Suntharasamai P, et al, for the Bangkok Tenofovir Study Group. Antiretroviral prophylaxis for HIV infection in injecting drug users in Bangkok,
Thailand (the Bangkok Tenofovir Study): a randomised, double-blind, placebocontrolled phase 3 trial. Lancet 2013:381:2083-90.

46 Jintarkanon S, Nakapiew S, Tien-udom N, et al. Unethical clinical trials in Thailand: a community response. Lancet 2005:365:1617-18.

47 Ukpong M, Peterson K. New HIV Vaccines and Microbicides Advocacy Society (NHVMAS) 2009; Oral Tenofovir Controversy II: Voices from the Field. http://www. nhvmas-ng.org

48 Page-Shafer K, Saphonn V, Sun LP, et al. HIV prevention research in a resourcelimited setting: the experience of planning a trial in Cambodia. Lancet 2005;366:1499-503.

49 Merson MH, Simonds RJ, Rogers MF. Ethics of placebo-controlled trials of zidovudine to prevent the perinatal transmission of HIV in the Third World. N Engl I Med 1998:338:836

50 De Zulueta P. Randomised placebo-controlled trials and HIV-infected pregnant women in developing countries. Ethical Imperialism or Unethical Exploitation. Bioethics 2001;15:289-311.

51 Lurie $\mathrm{P}$, Wolfe SM. Unethical trials of interventions to reduce perinatal transmission of the human immunodeficiency virus in developing countries. $N$ Engl I Med 1997:337:853-6.

52 Joint United Nations Programme on HIV/AIDS (UNAIDS). Ethical considerations in biomedical HIV prevention trials: UNAIDS/WHO Guidance Document. Secondary Ethical considerations in biomedical HIV prevention trials: UNAIDS/WHO Guidance Document. 2012. http://www.unaids.org/en/media/unaids/contentassets/documents/ unaidspublication/2012/jc1399_ethical_considerations_en.pdf (accessed May 2015).

53 Rennie S, the HPTN Ethics Working Group. HIV Prevention Trials Network Ethics Guidance for Research, Revised 10 June 2009. Secondary HIV Prevention Trials Network Ethics Guideance for Research, Revised 10 June 2009 http://hptn.org/web\% 20documents/EWG/HPTNEthicsGuidanceV10Jun2009.pdf

54 London A. The ambiguity and the exigency: clarifying 'standard of care' arguments in international research. J Med Philos 2000:25:379-97.

55 London A. Equipose and international human-subjects research. Bioethics 2001:15:312-32.

56 Dawson L, Zwerski S. Clinical trial design for HIV prevention research: determining standards of prevention. Bioethics 2015;29:316-23.

57 Haire B, Oluwatoyin Folayan M, Hankins C, et al. Ethical considerations in determining standard of prevention packages for HIV prevention trials: examining PrEP. Developing World Bioethics 2013;13:87-94. http://onlinelibrary.wiley.com/doi/ 10.1111/dewb.12032/full

58 Moorhouse R, Slack C, Quayle M, et al. Stakeholder views of ethical guidance regarding prevention and care in HIV vaccine trials. BMC Med Ethics 2014;15:51.

59 Macqueen KM, McLoughlin $\mathrm{K}$, Alleman $\mathrm{P}$, et al. Partnering for care in HIV prevention trials. J Empir Res Hum Res Ethics 2008;3:5-18.

60 Beyrer C, Malinowska-Sempruch K, Kamarulzaman A, et al. Time to act: a call for comprehensive responses to HIV in people who use drugs. Lancet 2010;376:551-63.

61 Irwanto DN, Praptorahario I, Irianto $S$, et al. on behalf of 11 signatories. Evidenceinformed response to illicit drugs in Indonesia. Lancet 2015;385:2249-50.

62 Wolfe D. Beyond the Hype: PrEP for People Who Inject Drugs. The Huffington Post [Internet] 201314 June. 2013. https://www.opensocietyfoundations.org/voices/ beyond-hype-prep-people-who-inject-drugs

63 Krupitsky E, Zvartau E, Woody G. Use of naltrexone to treat opioid addiction in a country in which methadone and buprenorphine are not available. Curr Psychiatry Rep 2010:12:448-53.

64 Hoffman IF, Latkin CA, Kukhareva PV, et al. A peer-educator network HIV prevention intervention among injection drug users: results of a randomized controlled trial in St. Petersburg, Russia. AIDS Behav 2013;17:2510-20. 